\title{
Three-dimensional nanoscale study of Al segregation and quantum dot formation in GaAs/AIGaAs core-shell nanowires
}

\author{
L. Mancini, ${ }^{1}$ Y. Fontana,${ }^{2}$ S. Conesa-Boj, ${ }^{2}$ I. Blum,${ }^{1}$ F. Vurpillot, ${ }^{1}$ L. Francaviglia ${ }^{2}$ \\ E. Russo-Averchi, ${ }^{2}$ M. Heiss, ${ }^{2}$ J. Arbiol, ${ }^{3,4}$ A. Fontcuberta i Morral, ${ }^{2}$ and L. Rigutti ${ }^{1, a)}$ \\ ${ }^{1}$ Groupe de Physique des Matériaux, UMR CNRS 6634, University and INSA of Rouen, Normandie University, \\ 76800 St. Etienne du Rouvray, France \\ ${ }^{2}$ Laboratoire des Matériaux Semiconducteurs, École Polytechnique Fédérale de Lausanne, 1015 Lausanne, \\ Switzerland \\ ${ }^{3}$ Institut de Ciència de Materials de Barcelona (ICMAB-CSIC), Campus de la UAB, 08193 Bellaterra, CAT, \\ Spain \\ ${ }^{4}$ Institució Catalana de Recerca i Estudis Avançats (ICREA), 08010 Barcelona, CAT, Spain
}

(Received 20 November 2014; accepted 7 December 2014; published online 17 December 2014)

GaAs/Al-GaAs core-shell nanowires fabricated by molecular beam epitaxy contain quantum confining structures susceptible of producing narrow photoluminescence (PL) and single photons. The nanoscale chemical mapping of these structures is analyzed in 3D by atom probe tomography (APT). The study allows us to confirm that $\mathrm{Al}$ atoms tend to segregate within the AlGaAs shells towards the vertices of the hexagons defining the nanowire cross section. We also find strong alloy fluctuations remaining AlGaAs shell, leading occasionally to the formation of quantum dots (QDs). The PL emission energies predicted in the framework of a 3D effective mass model for a QD analyzed by APT and the PL spectra measured on other nanowires from the same growth batch are consistent within the experimental uncertainties. (C) 2014 AIP Publishing LLC.

[http://dx.doi.org/10.1063/1.4904952]

Epitaxial semiconductor quantum dots (QDs) have been extensively studied over the last three decades because of their peculiar optical properties such as narrow luminescence and single photon emission. The structure and chemical composition of QDs has traditionally been assessed by scanning tunneling microscopy (STM) or transmission electron microscopy (TEM) related techniques. ${ }^{1}$ In the last decade, laser-assisted atom probe tomography (LA-APT) ${ }^{2}$ has emerged as a tool for the determination of the morphology and of the chemical composition of nanoscale semiconductor heterostructures with sub-nanometer resolution in $3 \mathrm{D} .^{3,4}$ In the past few years, several APT-based studies addressed a limited number of QD structures. ${ }^{4-10}$ Quantum dots can be inserted in semiconductor nanowires either deterministically, with specific shape and composition, ${ }^{11-14}$ or as self-assembled structures. ${ }^{15-18}$ Nanowires, furthermore, represent a model system for atom probe analysis, ${ }^{19-24}$ as they closely approximate the shape of a field-emission tip. Nevertheless, and despite the interest of nanowire-based QDs for quantum information $^{25-29}$ and for their integration in nanoscale optoelectronic devices, ${ }^{30-33}$ no studies of nanowire QDs by APT have been reported yet.

Core-shell GaAs/AlGaAs nanowires grown by molecular beam epitaxy (MBE) have been shown to exhibit narrow luminescence and single photon emission, ascribed to QDs forming occasionally at the external corners of the AlGaAs shells. ${ }^{27}$ Isolated emissions centered around $670 \mathrm{~nm}(\sim 1.85 \mathrm{eV})$ could be found by cathodoluminescence (CL) spectroscopy, with a typical mean frequency along the axis of the order of one spot per micron. ${ }^{27}$

\footnotetext{
${ }^{\text {a) }}$ Author to whom correspondence should be addressed. Electronic mail: lorenzo.rigutti@univ-rouen.fr
}

Previous studies of these structures performed by highresolution scanning transmission electron microscopy (HR-STEM), electron energy loss spectroscopy (EELS), and energy-dispersive X-ray spectroscopy (EDX) indicated that QDs may form at the outer corner of the hexagon in the AlGaAs shell, at the termination of an $\mathrm{Al}$-segregation region. ${ }^{27,34}$ However, it was not possible to fully characterize the shape of the dots nor ascertain whether other mechanisms of QD formation may occur within the AlGaAs shell. In addition, the elemental contrast in TEM techniques is limited by the thickness of the slab and by the density contrast of the nanostructure.

In this work, we performed a 3D APT study on GaAs/ AlGaAs core-shell nanowires, finding evidence for QD formation within the AlGaAs shell. One such dot, found in the volume of a nanowire sample, could be reconstructed in its 3D shape and composition: it is elongated in the direction of the nanowire axis and is defined by a composition gradient of the AlGaAs alloy. Such QD structures are thus extremely elusive with respect to STEM investigation. Their optical properties have been studied experimentally by microphotoluminescence $(\mu$-PL) and theoretically by solving the Schrödinger equation in the effective mass approximation based directly on the alloy distribution issued by the atom probe data. The expected transition energy is consistent with the statistics of the excitonic QD emission collected from a set of nanowires from the same growth batch.

GaAs/AlGaAs core/shell nanowire structures were grown in a P600 DCA MBE system. The GaAs core was grown by the Ga-assisted method on a (111) silicon wafer, with conditions optimized for a high yield of vertical wires. ${ }^{35}$ After the nanowires reached around a $10 \mu \mathrm{m}$ length, the gallium flux was stopped, the As4 flux increased, and the 

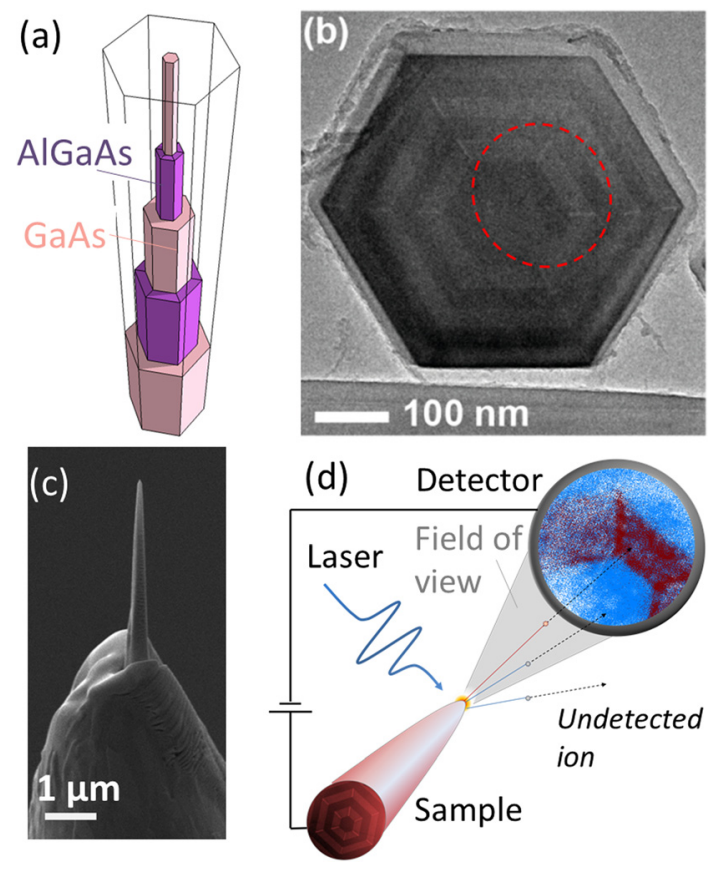

FIG. 1. (a) Schematics and (b) cross-sectional TEM image illustrating the shape and the internal structure of the studied NWs. The red dashed circle indicates schematically the zone analyzed by atom probe in sample NW3. (c) Field emission tip obtained after FIB annular milling of a nanowire. (d) Schematics of the atom probe experiment.

temperature lowered to $460{ }^{\circ} \mathrm{C}$. The growth of a shell with nominally $33 \%$ of Al followed. See supplementary material at Ref. 36 and previous Refs. 27 and 37 for more details on the exact conditions. The scheme of the core-shell structure is reported in Fig. 1(a), while a bright-field low magnification STEM image of a cross section of a nanowire, prepared by diamond lapping film mechanical thinning followed by precision ion polishing (PIPS) ${ }^{37,38}$ is reported in Fig. 1(b), the AlGaAs and GaAs shells appearing in brighter and darker contrast, respectively.

Nanowires could be analyzed by APT after being sharpened by focused ion beam, as shown in Fig. 1(c). The APT experiment is schematically illustrated in Fig. 1(d). The nanowires were analyzed in a Cameca laser-assisted wideangle tomographic atom probe (LAWATAP), having a field of view (i.e., the half-angle of the detector acceptance cone) of $\alpha=20^{\circ}$. Due to this geometrical constraint and to the large wire diameter, APT allows for studying only a part of the innermost AlGaAs shell. A total of four nanowires, labelled NW1-4, were analyzed by APT. See supplementary material at Ref. 36 for the details of sample preparation and for the specific APT analysis conditions.

Data issued by the 3D APT analysis of NW3 are reported in Fig. 2. Notice that the size of the analyzed region does not exactly correspond to the scheme in Fig. 1(b), due to the dispersion in size between different nanowires. Figure 2(a) reports in blue the isoconcentration surfaces defined for a threshold $\mathrm{Al}$ atomic concentration equal to $4 \%$, while Fig. 2(b) reports in red the $21 \% \mathrm{Al}$ atomic concentration isosurfaces. Both isosurfaces, a subset of $5 \%$ of the Al atom positions and selected regions of interest (ROI) are also displayed in the top view of Fig. 2(c). The imaged volume is a part of the innermost AlGaAs shell, which is delimited by the blue isosurfaces in Figs. 2(a) and 2(c). Parts of the GaAs core and of an intermediate GaAs shell are also visible. The definition of the interface between the AlGaAs shell and the outer GaAs shell has been analyzed in the ROI \#1. (a)

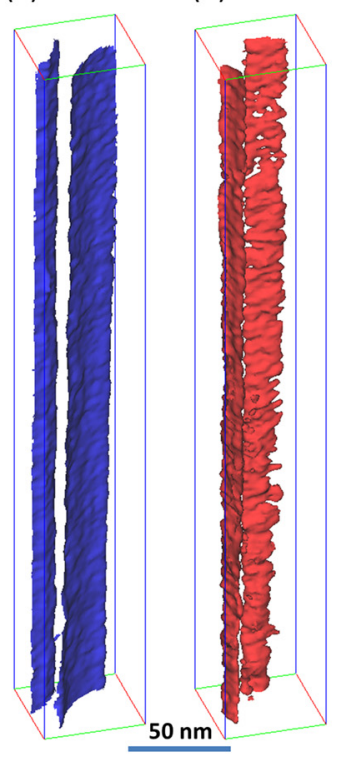

(c)

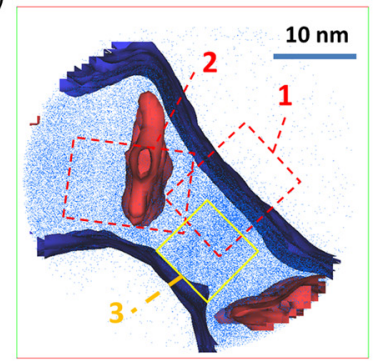

(f)

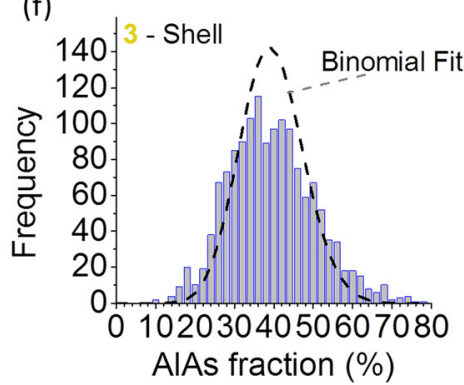

(d)

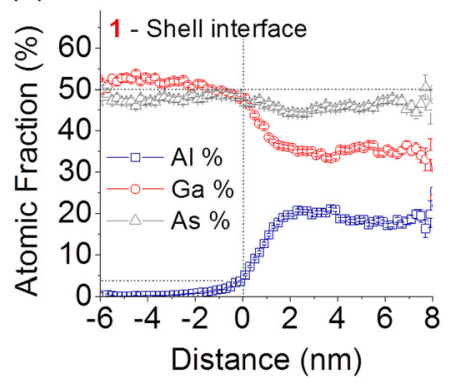

(e)

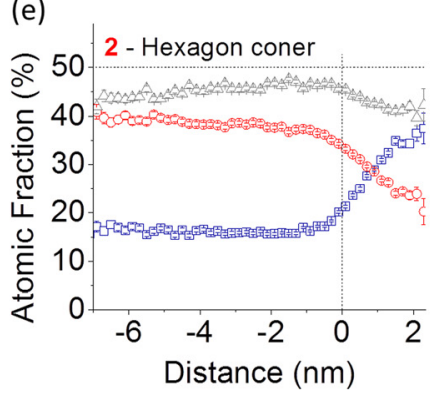

FIG. 2. Atom probe 3D reconstruction of the volume analyzed from sample NW3, showing side views of the isoconcentration surfaces defined for Al atomic concentration thresholds of 4\% ((a), blue) delimiting the AlGaAs shell and of $21 \%$ ((b), red) delimiting the Al segregation regions at the hexagon corner lines. (c) Top view of the reconstructed volume, reporting the isosurfaces at $4 \%$ (blue) and $21 \% \mathrm{Al}$ elemental concentration (red); a $5 \%$ of the $\mathrm{Al}$ atoms is also reported as blue dots. The ROI \#1-3 relate to the analyses displayed in (d)-(f), respectively. (d) and (e) Proximity histograms defining the elemental concentrations of Al (blue squares), Ga (red circles), and As (grey triangles) in the ROI \#1 and \#2, respectively. (f) AlAs alloy distribution extracted from ROI \#3, binned into volume elements containing 100 ions each. ROI \#1-2 are as deep as the whole analyzed volume. ROI \#3 is 50 nm deep and centered at around $150 \mathrm{~nm}$ from the top of the large reconstruction volume. 
The result is reported in Fig. 2(d) as a proximity histogram $\left(\right.$ or proxigram ${ }^{39}$ ) defined for a $4 \% \mathrm{Al}$ atomic concentration isosurface. The proxigram indicates an abrupt variation of the $\mathrm{Al}$ atomic concentration from $0 \%$ in the GaAs shell to about 20\% in the AlGaAs shell. The data evidenced in Figs. 2(b) and 2(c) indicate that $\mathrm{Al}$ tends to segregate along the (101) planes crossing the vertices of the hexagon. Al segregation has been previously observed by cross-sectional STEM, as visible in Fig. 1(b). Segregation occurs during the growth due to the differences in chemical potential and adatom mobility on the (111)- and (112)-type facets. ${ }^{27}$ It has also been observed in other Al-containing hexagonal nanowire structures. ${ }^{40-42}$ The proxigram defined in the ROI \#2 for an $\mathrm{Al} 21 \%$ isosurface is displayed in Fig. 2(e), and shows that these $\mathrm{Al}$ segregation planes contain up to $40 \% \mathrm{Al}$ atomic concentration, with an interface defined over about $2 \mathrm{~nm}$. It is interesting to notice that the detected As fraction is reasonably close to $50 \%$ in the GaAs shell, but rather closer to $45 \%$ in the AlGaAs shell, and even to $40 \%$ in the AlGaAs segregation region. It is important to underline that this deviation from the nominal III-V stoichiometric ratio does not correspond to a compositional bias within the crystal, but is an artefact of the measurement, due to different field-dependent evaporation efficiencies of $\mathrm{Ga}$ and As, and to the different average evaporation field of the GaAs and of the AlGaAs phases, as previously studied in other wide bandgap semiconductors. ${ }^{43-45}$ This small but not negligible discrepancy has no particular consequences for a correct quantification of the $\mathrm{x}$ fraction of $\mathrm{AlAs}$ in $\mathrm{Al}_{\mathrm{x}} \mathrm{Ga}_{1-\mathrm{x}} \mathrm{As}$, if this is determined as the ratio of $\mathrm{Al}$ to $(\mathrm{Al}+\mathrm{Ga})$ atomic concentrations. ${ }^{45}$

Finally, the frequency histogram shown in Fig. 2(f) and defined for the ROI \#3, including a sub-volume of the
AlGaAs shell outside of the segregation planes, also indicates that the AlAs alloy fraction exhibits strong fluctuations and does not follow the binomial distribution expected for a random alloy.

The analysis of the interface between the inner $\mathrm{AlGaAs}$ shell and the subsequent GaAs shell close to the corner of the hexagon evidences alloy fluctuations and interface roughness, but no definite features that could be attributed to QDs such as in Refs. 27 and 34. See supplementary material at Ref. 36 for the details. This result is related with the low frequency of occurrence of such QDs along the axis and the small volume probed by APT: a single atom probe run typically investigates a depth of some hundred nanometers visualizing only one or two vertices of the AlGaAs shell.

Evidence for QD formation was found at a different location, namely, in the AlGaAs shell outside of the Al segregation plane. This result is shown in Fig. 3. Fig. 3(a) reports the isosurfaces defined for a threshold AlAs alloy fraction equal to $14 \%$ in a $3 \mathrm{D}$ reconstructed sub-volume of sample NW3. In the shown perspective, the AlGaAs shell lies in the central vertical stripe between the isosurfaces, while the GaAs core (outer shell) lies in the left-hand (righthand) stripe. The nearly spherical isosurface with a diameter of $\sim 4 \mathrm{~nm}$ in the center of the graph contains an Al-depleted region, where the AlAs alloy fraction is lower than $14 \%$. A 2D AlAs fraction map defined over a $1 \mathrm{~nm}$ thick slice crossing the QD is reported in Fig. 3(b). Furthermore, Fig. 3(c) and a supplementary video (Multimedia view) visualize the 3D distribution of the AlAs alloy fraction in a subvolume of the AlGaAs shell containing the QD. These data indicate that the QD is elongated in the direction of the nanowire axis, and that it is defined by a gradient of AlGaAs composition.

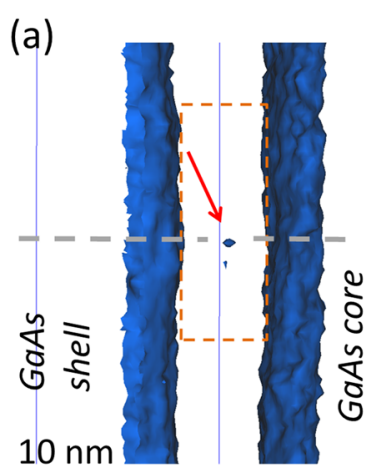

(c)

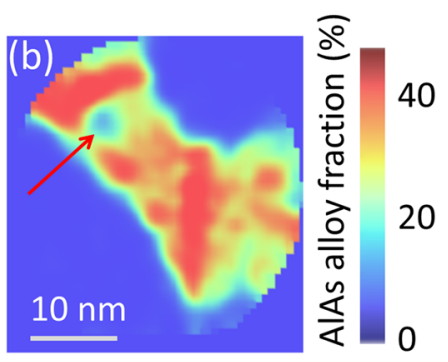

40 (d)
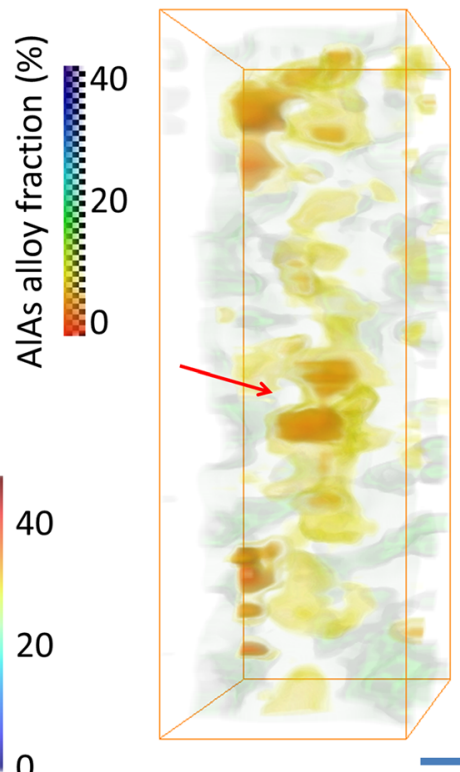

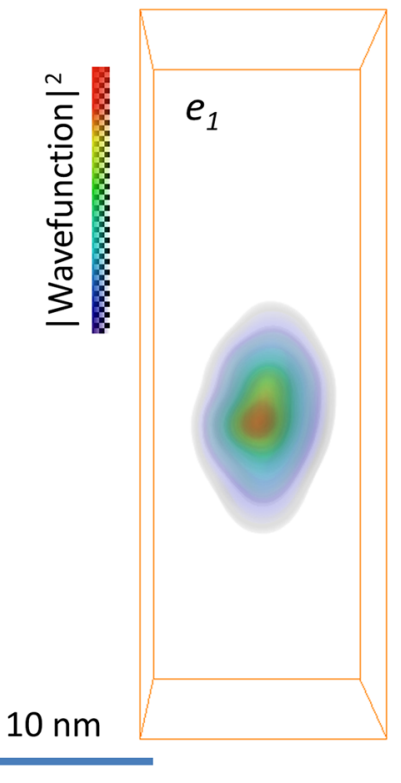

(e)

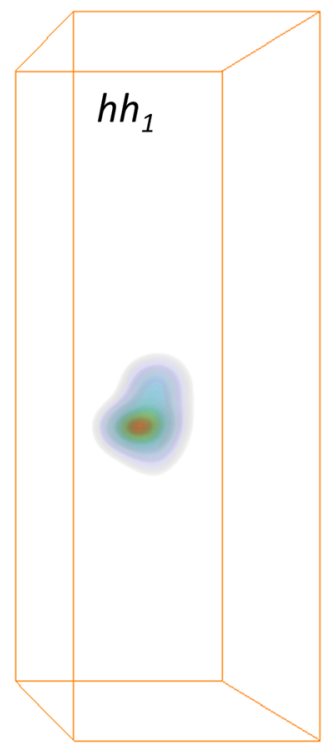

FIG. 3. (a) Atom probe 3D reconstruction of the sub-volume of sample NW3 containing a QD in the AlGaAs shell, showing the isosurfaces at 14\% AlAs alloy fraction; the GaAs core and the external GaAs shell appear on the right and on the left-hand side, respectively; (b) 2D map of Al elemental concentration, extracted from the $1 \mathrm{~nm}$ thick slice corresponding to the grey dashed line in (a). The red arrows point to the QD position. (c) 3D visualization of the AlAs alloy fraction distribution in the region of interest highlighted in (a). (The low Al density regions on the edges correspond to the superposition of the ROI with the GaAs shell). (d) and (e) Wavefunctions of the single-particle ground states of electron (d) and heavy hole (e) calculated in the framework of an effective mass approximation for the alloy distribution in (c). See also the 3D animated graphics of (c)-(e). (Multimedia view) [URL: http://dx.doi.org/10.1063/1.4904952.1] 


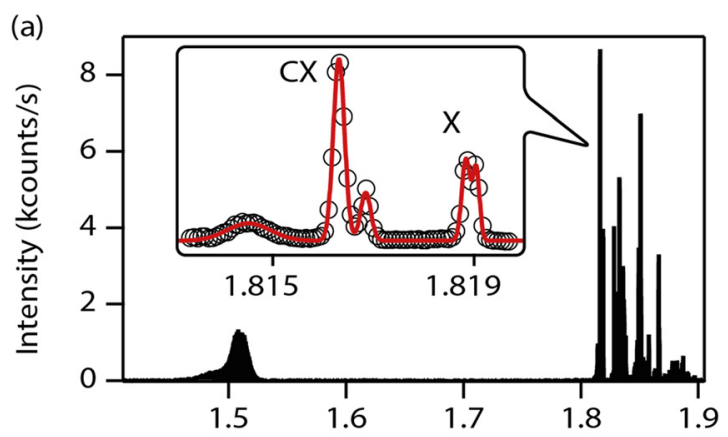

(b)

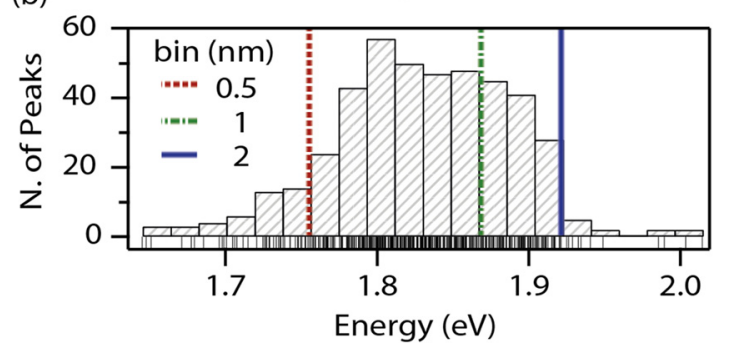

FIG. 4. (a) A typical $\mu \mathrm{PL}$ spectrum obtained from a nanowire issued from the same growth batch; (b) Histogram of the excitonic emission energies collected after the analysis of 27 spectra from the same growth batch. The position of single peaks is reported in the lower part of the graph. Superimposed, the transition energies for the single particle states $e_{1}-h_{1}$ calculated within the effective mass approximation for the QD system shown in Fig. 3 and for different choices of binning width for the definition of the local AlAs fraction.

Along the analyzed volume of sample NW3 and in the other samples, no other alloy fluctuations of similar strength were found.

The optical properties of nanowires from the same growth batch are displayed in Fig. 4. The PL was collected at a temperature of $3.85 \mathrm{~K}$ upon stabilized continuous-wave (CW) excitation at $1.958 \mathrm{eV}$, providing a resolution of $\sim 500 \mathrm{~nm}$ and a power density of $\sim 650 \mathrm{~W} \mathrm{~cm}^{-2}$ (values for a focus on flat $\mathrm{Si}$ ). The signal was analyzed by a $1200 \mathrm{l} / \mathrm{mm}$ grating in a $500 \mathrm{~mm}$ spectrometer giving a spectral resolution of $80 \mu \mathrm{eV}$, projected and read-out on a LN2-cooled silicon charged-coupled device (CCD). Scanning the grating allowed to record a high-resolution spectrum on a large bandwidth. A typical single-QD $\mu \mathrm{PL}$ spectrum is displayed in Fig. 4(a). The full spectrum exhibits a broad emission peak at $\sim 1.5 \mathrm{eV}$, corresponding to the GaAs core and narrow emission lines at higher energies. The inset shows the spectral interval centered around $1.817 \mathrm{eV}$, where emission lines emitted by a single QD are found. The blue edge peak (labelled $\mathrm{X}$ ) corresponds to excitonic emission $\left(1 e_{1}+1 h h_{l}\right)$. The effect of the electron-hole exchange interaction can be seen in the small splitting of the peak. The red-shifted peak (labelled CX) can be attributed to the same QD in a charged configuration. Emerging peaks around $1.814 \mathrm{eV}$ and $1.817 \mathrm{eV}$ correspond, respectively, to the biexciton (broadened by its sideband ${ }^{46}$ ) and the second charged exciton. ${ }^{34}$

The histogram of the QD single exciton transition energies issued by the analysis of the same batch of nanowires used for APT is shown in Fig. 4(b). In this case, the spectra were obtained on samples cooled at $10 \mathrm{~K}$, upon $\mathrm{CW}$ excitation at $2.54 \mathrm{eV}$, with a power density around $150 \mathrm{~W} \mathrm{~cm}^{-2}$. The signal was dispersed with a $300 \mathrm{l} / \mathrm{mm}$ grating and projected on a
Peltier cooled CCD. Around 440 peaks from 27 different spectra were located by a peak recognition algorithm.

In order to determine if the QD highlighted in Figs. 3(a)-3(c) is consistent with the optical spectroscopy, the results of the $3 \mathrm{D}$ elemental mapping were used to build the potential landscape for calculation of the electronic states. These were determined in the framework of an effective-mass approximation implemented by the software nextnano ${ }^{3}$ (Ref. 47) involving the computation of the single-particle states for the conduction and heavy-hole states ${ }^{48}$ in a structure assumed as elastically relaxed. Since the computed value corresponds to a single point in the statistical average of ensemble, a more sophisticated technique is not primordial.

The single-particle electron and heavy hole $\left(e_{1}-h h_{1}\right)$ squared wavefunctions are displayed in Figs. 3(d) and 3(e), while the associated single particle-state transition energies are superimposed to the histogram of Fig. 4(b). It is evident that the result of the calculation is critically dependent on the choice of the size of the sampling volume element over which the local AlAs alloy fraction is defined, while the exciton binding energy, not displayed, is rather constant around $30 \mathrm{meV}$. If, on one hand, it is not reasonable to adopt a bin width larger than $2 \mathrm{~nm}$ - the width of the region containing less than $14 \%$ AlAs within the dot is around $3 \mathrm{~nm}$ only-it is also not meaningful to decrease the binning width below $0.5 \mathrm{~nm}$, because (i) this value approximately corresponds to the lateral resolution of the measurement of single atom positions in atom probe and (ii) such small bins make the statistical definition of the composition not reliable. The results of the simulations indicate thus that the emission energies for the QD measured in APT should be found within the interval 1.75-1.92 eV. According to these considerations, the structural properties of the QD found by APT are well consistent with the optical properties of the excitonic QD emission found on other nanowires from the same series.

In summary, we have performed a comparative measurement between the structural and optical properties of core-shell GaAs/AlGaAs nanowires. The three-dimensional APT analysis indicates that AlGaAs shells exhibit Al-rich segregation planes along the (101) planes and a non-random alloy distribution in the rest of the shell. Alloy fluctuations of such strength may induce the formation of QDs-an example of which was found in the proximity of the Al-rich segregation plane. The Schrödinger equation in the effective mass approximation was subsequently solved for the alloy distribution measured by APT, yielding QD emission energies consistent with the $\mu \mathrm{PL}$ study performed on other nanowire samples from the same growth batch.

This study opens up interesting perspectives, as it will be possible, in the next future, to address the correlation between the optical and structural properties of single QDs, provided the optical spectroscopy and atom probe tomography studies are performed subsequently on the same nanoscale object. $^{49}$

The authors from GPM Rouen (L.M., I.B., F.V., and L.R.) acknowledge funding through projects EMC3 Labex ASAP and ANR-13-JS10-0001-01 Tapoter. The authors from EPFL Lausanne (Y.F., S.C.B., L.F., E.R.A., and A.F.i.M.) thank funding from SNF through the NCCR QSIT and the 
DACH program via the project Nanowiring (No. 134506). J.A. acknowledges funding from Generalitat de Catalunya 2014 SGR 1638. Authors acknowledge F. J. Belarre for TEM sample preparation.

${ }^{1}$ V. A. Shchukin and D. Bimberg, Rev. Mod. Phys. 71, 1125 (1999).

${ }^{2}$ B. Gault, F. Vurpillot, A. Vella, M. Gilbert, A. Menand, D. Blavette, and B. Deconihout, Rev. Sci. Instrum. 77, 043705 (2006)

${ }^{3}$ T. F. Kelly, D. J. Larson, K. Thompson, R. L. Alvis, J. H. Bunton, J. D. Olson, and B. Gorman, Annu. Rev. Mater. Sci. 37, 681 (2007).

${ }^{4}$ M. Müller, A. Cerezo, G. D. W. Smith, L. Chang, and S. S. A. Gerstl, Appl. Phys. Lett. 92, 233115 (2008).

${ }^{5}$ B. P. Gorman, A. G. Norman, and Y. Yan, Microsc. Microanal. 13, 493-502 (2007).

${ }^{6}$ A. D. Giddings, J. G. Keizer, M. Hara, G. J. Hamhuis, H. Yuasa, H. Fukuzawa, and P. M. Koenraad, Phys. Rev. B 83, 205308 (2011).

${ }^{7}$ A. J. Martin, J. Hwang, E. A. Marquis, E. Smakman, T. W. Saucer, G. V. Rodriguez, A. Hunter, V. Sih, P. M. Koenraad, J. D. Phillips, and J. Mirecki, Appl. Phys. Lett. 102, 113103 (2013).

${ }^{8}$ A. Maryński, G. Sek, A. Musiał, J. Andrzejewski, J. Misiewicz, C. Gilfert, J. P. Reithmaier, A. Capua, O. Karni, D. Gready et al., J. Appl. Phys. 114, 094306 (2013).

${ }^{9}$ H. Benallali, T. Cremel, K. Hoummada, D. Mangelinck, R. André, S. Tatarenko, and K. Kheng, Appl. Phys. Lett. 105, 053103 (2014).

${ }^{10}$ J. Bocquel, A. D. Giddings, T. Mano, T. J. Prosa, D. J. Larson, and P. M. Koenraad, Appl. Phys. Lett. 105, 153102 (2014).

${ }^{11}$ M. T. Borgström, V. Zwiller, E. Müller, and A. Imamoglu, Nano Lett. 5, 1439-1443 (2005).

${ }^{12}$ M. Tchernycheva, G. E. Cirlin, G. Patriarche, L. Travers, V. Zwiller, U. Perinetti, and J. C. Harmand, Nano Lett. 7, 1500-1504 (2007).

${ }^{13}$ G. Bulgarini, M. E. Reimer, T. Zehender, M. Hocevar, E. P. Bakkers, L. P. Kouwenhoven, and V. Zwiller, Appl. Phys. Lett. 100, 121106 (2012).

${ }^{14}$ A. Pierret, M. Hocevar, S. L. Diedenhofen, R. E. Algra, E. Vlieg, E. C. Timmering, M. A. Verschuuren, G. W. G. Immink, M. A. Verheijen, and E. P. A. M. Bakkers, Nanotechnology 21, 065305 (2010).

${ }^{15}$ E. Uccelli, J. Arbiol, J. R. Morante, and A. Fontcuberta i Morral, ACS Nano 4, 5985-5993 (2010).

${ }^{16}$ L. Wang, A. Rastelli, S. Kiravittaya, P. Atkinson, F. Ding, C. B. Bufon, C. Hermannstädter, M. Witzany, G. J. Beirne, P. Michler, and O. G. Schmidt, New J. Phys. 10, 045010 (2008).

${ }^{17}$ E. Uccelli, M. Bichler, S. Nürnberger, G. Abstreiter, and A. Fontcuberta i Morral, Nanotechnology 19, 045303 (2008).

${ }^{18}$ Q. Zhu, F. Karlsson, A. Rudra, E. Pelucchi, and E. Kapon, Physica E 40, 1815-1818 (2008).

${ }^{19}$ W. H. Chen, V. G. Dubrovskij, X. Liu, T. Xu, R. Lardé, J. P. Nys, B. Grandidier, D. Stiévenard, G. Patriarche, and P. Pareige, J. Appl. Phys. 111, 094909 (2012).

${ }^{20}$ W. Chen, L. Yu, F. Z. S. Misra, P. Pareige, G. Patriarche, and P. Roca i Cabarrocas, Nat. Commun. 5, 4134 (2014).

${ }^{21}$ D. Perea, J. A. Allen, S. J. May, B. W. Wessels, D. N. Seidman, and L. J. Lauhon, Nano Lett. 6, 181 (2006).

${ }^{22}$ D. Perea, E. R. Hemesath, E. J. Schwalbach, J. L. Lensch-Falck, P. W. Voorhees, and L. J. Lauhon, Nat. Nanotechnol. 4, 315 (2009).

${ }^{23}$ O. Moutabnabbir, D. Isheim, H. Blumtritt, S. Senz, E. Pippel, and D. Seidman, Nature 496, 78 (2013).

${ }^{24}$ S. Du, T. Burgess, B. Gault, Q. Gao, P. Bao, L. Li, X. Cui, W. K. Yeoh, H. Liu, L. Yao et al., Ultramicroscopy 132, 186-192 (2013).

${ }^{25}$ M. E. Reimer, G. Bulgarini, N. Akopian, M. Hocevar, M. Bouwes Bavinck, M. A. Verheijen, E. P. A. M. Bakkers, L. P. Kouwenhoven, and V. Zwiller, Nat. Commun. 3, 737 (2012).
${ }^{26}$ M. H. van Weert, N. Akopian, U. Perinetti, M. P. van Kouwen, R. E. Algra, M. A. Verheijen, E. P. A. M. Bakkers, L. P. Kouwenhoven, and V. Zwiller, Nano Lett. 9, 1989-1993 (2009).

${ }^{27}$ M. Heiss, Y. Fontana, A. Gustafsson, G. Wüst, C. Magen, D. D. O'Regan, J. W. Luo, B. Ketterer, S. Conesa-Boj, A. V. Kuhlmann et al., Nat. Mater. 12, 439-444 (2013).

${ }^{28}$ M. A. Versteegh, M. E. Reimer, K. D. Jöns, D. Dalacu, P. J. Poole, A. Gulinatti, A. Giudice, and V. Zwiller, Nat. Commun. 5, 5298 (2014).

${ }^{29}$ T. Huber, A. Predojević, M. Khoshnegar, D. Dalacu, P. J. Poole, H. Majedi, and G. Weihs, Nano Lett. 14, 7107 (2014).

${ }^{30}$ M. P. Van Kouwen, M. H. M. Van Weert, M. E. Reimer, N. Akopian, U. Perinetti, R. E. Algra, E. P. A. M. Bakkers, L. P. Kouwenhoven, and V. Zwiller, Appl. Phys. Lett. 97, 113108 (2010).

${ }^{31}$ M. T. Bjork, B. J. Ohlsson, C. Thelander, A. I. Persson, K. Deppert, L. R. Wallenberg, and L. Samuelson, Appl. Phys. Lett. 81, 4458 (2002).

${ }^{32}$ E. D. Minot, F. Kelkensberg, M. Van Kouwen, J. A. Van Dam, L. P. Kouwenhoven, and V. Zwiller, Nano Lett. 7, 367 (2007).

${ }^{33}$ L. Rigutti, M. Tchernycheva, A. De Luna Bugallo, G. Jacopin, F. H. Julien, K. March, O. Stephan, M. Kociak, and R. Songmuang, Nano Lett. 10, 2939 (2010)

${ }^{34}$ Y. Fontana, P. Corfdir, B. Van Hattem, E. Russo-Averchi, M. Heiss, S. Sonderegger, C. Magen, J. Arbiol, R. T. Phillips, and A. Fontcuberta i Morral, Phys. Rev. B 90, 075307 (2014).

${ }^{35}$ E. Russo-Averchi, M. Heiss, L. Michelet, P. Krogstrup, J. Nygard, C. Magen, J. R. Morante, E. Uccelli, J. Arbiol, and A. Fontcuberta i Morral, Nanoscale 4, 1486-1490 (2012).

${ }^{36}$ See supplementary material at http://dx.doi.org/10.1063/1.4904952 for details on sample growth, sample preparation for Atom Probe Tomography (APT), APT experimental details, and APT analysis of other nanowire samples.

${ }^{37}$ A. Fontcuberta i Morral, D. Spirkoska, J. Arbiol, M. Heigoldt, J. R. Morante, and G. Abstreiter, Small 4, 899-903 (2008).

${ }^{38}$ M. Heigoldt, J. Arbiol, D. Spirkoska, J. M. Rebled, S. Conesa-Boj, G. Abstreiter, F. Peiró, J. R. Morante, and A. Fontcuberta i Morral, J. Mater. Chem. 19, 840-848 (2009).

${ }^{39}$ O. C. Hellman, J. A. Vandenbroucke, J. Rüsing, D. Isheim, and D. N. Seidman, Microsc. Microanal. 6, 437-444 (2000).

${ }^{40}$ N. Sköld, J. B. Wagner, G. Karlsson, T. Hernán, W. Seifert, M. E. Pistol, and L. Samuelson, Nano Lett. 6, 2743-2747 (2006).

${ }^{41}$ C. Durand, C. Bougerol, J. F. Carlin, G. Rossbach, F. Godel, J. Eymery, P.-H. Jouneau, A. Mukhtarova, R. Butté, and N. Grandjean, ACS Photonics 1, 38-46 (2014).

${ }^{42}$ J. Arbiol, C. Magen, P. Becker, G. Jacopin, A. Chernikov, S. Schäfer, F. Furtmayr, M. Tchernycheva, L. Rigutti, J. Teubert et al. Nanoscale 4, 7517-7524 (2012).

${ }^{43}$ J. Riley, R. A. Bernal, Q. Li, H. D. Espinosa, G. T. Wang, and L. J. Lauhon, ACS Nano 6, 3898 (2012).

${ }^{44}$ J. R. Riley, T. Detchprohm, C. Wetzel, and L. J. Lauhon, Appl. Phys. Lett. 104, 152102 (2014).

${ }^{45}$ L. Mancini, N. Amirifar, D. Shinde, I. Blum, M. Gilbert, A. Vella, F. Vurpillot, W. Lefebvre, R. Lardé, E. Talbot et al., J. Phys. Chem. C 118, 24136-24151 (2014).

${ }^{46}$ P. Senellart, E. Peter, J. Hours, A. Cavanna, and J. Bloch, Phys. Rev. B 72, 115302 (2005).

${ }^{47}$ S. Birner, see http://www.nextnano.de/nextnano3/ for "Nextnano3" (last accessed September 19, 2014).

${ }^{48}$ M. Sabathil, "Opto-electronic and quantum transport properties of semiconductor nanostructures," Ph.D. thesis (Munich, 2005).

${ }^{49}$ L. Rigutti, I. Blum, D. Shinde, D. Hernàndez-Maldonado, W. Lefebvre, J. Houard, F. Vurpillot, A. Vella, M. Tchernycheva, C. Durand et al., Nano Lett. 14, 107-114 (2014). 